\title{
ON THE ALEXANDER POLYNOMIAL OF A CYCLICALLY PERIODIC KNOT
}

\author{
JONATHAN A. HILLMAN
}

\begin{abstract}
We show that a theorem of Burde on the Alexander polynomial of a cyclically periodic knot $K$, such that $\Delta_{2}(K)=1$, may be extended to all cyclically periodic knots.
\end{abstract}

A knot $K$ in a homology 3-sphere $\Sigma$ has cyclic period $q$ if it is invariant under an automorphism of $\Sigma$ of order $q$ which has fixed point set a circle disjoint from $K$. In [3] we gave new proofs of theorems of Burde [1] and Murasugi [5] on the Alexander polynomial $\Delta_{1}(K)$ of such a knot.

A refinement of the second lemma of [3] enables us to remove the restriction $\Delta_{2}(K)=1$ from Burde's theorem. We shall adopt the notation of [3].

THEOREM. Let $K$ be a knot in an homology 3-sphere $\Sigma$ which has cyclic period $q$, where $q=p^{r}$ for some prime $p$. Then either

(i) $\Delta_{1}(K) \equiv 1$ modulo $(p)$ or

(ii) the degree of the qth roots of unity over the splitting fields of $\Delta_{1}(K)$ is at most $n$, where $\Delta_{n}(K) \neq 1$ but $\Delta_{n+1}(K)=1$.

Proof. The argument given in [3] shows that if (i) fails then the $Q\left[t, t^{-1}\right]$-module $C=\left(G^{\prime} / G^{\prime \prime}\right) \otimes_{\mathbf{z}} Q$ (the rational homology of the infinite cyclic cover of the knot complement) has an automorphism of order exactly $q$. (For otherwise the $(q / p)$ th power of the given periodic self-homeomorphism $\tau$ of $\Sigma$ induces the identity on $G^{\prime} / G^{\prime \prime}$. Applying Milnor duality and the Wang sequence with coefficients $\mathbf{Z} / p \mathbf{Z}$ to the $p$-fold branched cyclic covering map from $Z$ to $\bar{Z}$, the results of 0 -framed surgery on $K$ in $\Sigma$ and on the image of $K$ in $\Sigma / \tau^{(q / p)}$, respectively, we conclude that $\left(G^{\prime} / G^{\prime \prime}\right) \otimes(\mathbf{Z} / p \mathbf{Z})$ must be 0 , and so $\Delta_{1}(K) \equiv 1$ modulo $(p)$.) Then for some irreducible factor $\delta$ of $\Delta_{1}(K)$, the $\delta$-primary submodule of $C$ has an automorphism $\tau$ of order exactly $q$, so we may assume $\Delta_{0}(C)=\delta^{l}$. Let $L=Q\left[t, t^{-1}\right] /(\delta)$. If $m \geqslant 1$ the kernel of the natural map from $\operatorname{Aut}\left(C / \delta^{m+1} C\right)$ to $\operatorname{Aut}\left(C / \delta^{m} C\right)$ is isomorphic to $\operatorname{Hom}\left(C / \delta^{m+1} C, \delta^{m} C / \delta^{m+1} C\right)$ and so is an $L$-vector space. In particular, it is torsion free. Therefore the image of $\tau$ in $\operatorname{Aut}(C / \delta C) \approx \mathrm{GL}(n, L)$ has order exactly $q$. Let $A=L[\tau]$ be the subalgebra of $M(n, L)$ generated by this element. Then $\operatorname{dim}_{L} A \leqslant n$ by the Cayley-Hamilton theorem. On the other hand, the image of $\tau$ in the semisimple algebra $A / \operatorname{rad} A$ again has order exactly $q$, as the kernel of the map on

Received by the editors December 15, 1981.

1980 Mathematics Subject Classification. Primary 57M25.

Key words and phrases. Alexander polynomial, cyclic period, knot, root of unity, splitting field.

(C)1983 American Mathematical Society $0002-9939 / 82 / 0000-2055 / \$ 01.50$ 
units is again an iterated extension of $L$-vector spaces. The algebra $A / \operatorname{rad} A$ is a product of fields containing $L$, so some factor is an extension $L^{\prime}$ of $L$ which contains the $q$ th roots of unity and for which $\left[L^{\prime}: L\right]=\operatorname{dim}_{L} L^{\prime} \leqslant \operatorname{dim}_{L} A \leqslant n$. Since $L$ is contained in the splitting field for $\Delta_{1}(K)$, the theorem follows.

REMARKs. Suppose (ii) holds. If $q=p^{r}$, with $r>1$, then either $L=L^{\prime}$, and so contains the $q$ th roots of unity, or $\left[L^{\prime}: L\right] \geqslant p$, and so $p \leqslant n$. If $q=p$ and $L$ is linearly disjoint from the field $Q(\zeta)$ of $p$ th roots of unity, that is if $L \cap Q(\zeta)=Q$, then $\left[L^{\prime}: L\right]=p-1$, so $p \leqslant n+1$.

If $\Delta_{2}(K)=1$, so that $n=1$, we obtain Burde's theorem [1] (itself an extension of a result of Trotter [8]). Burde's version applies to all but four of the prime knots with ten crossings or less (excepting $8_{18}, 9_{40}, 10_{99}$ and $10_{123}$ ), but does not usefully apply to the $n$-fold connected sum of a knot $K$ with itself, for $\Delta_{2}\left(\#^{n} K\right)$ is nontrivial unless $\Delta_{1}(K)=1$ or $n=1$.

For other recent work on knots with cyclic periods see $[\mathbf{2}, \mathbf{3}, \mathbf{4}, \mathbf{6}, \mathbf{7}]$. (Note that there are some discrepancies between the tables of [4] and [6].)

\section{REFERENCES}

1. G. Burde, Üher periodische knoten, Arch. Math. (Basel) 30 (1978), 487-492.

2. C. McA. Gordon, R. A. Litherland and K. Murasugi, Signatures of covering links, Canad. J. Math. 33 (1981), 381-394.

3. J. A. Hillman, New proofs of two theorems on periodic knots, Arch. Math. (Basel) 37 (1981), 457-461.

4. U. Lüdicke, Zyklische knoten, Arch. Math. (Basel) 32 (979), 588-599.

5. K. Murasugi, On periodic knots, Comment. Math. Helv. 46 (1971), 162-174.

6. On symmetry of knots, Tsukuba J. Math. 4 (1980), 331-347.

7. M. Sakuma, On the polynomials of periodic links, Math. Ann. 257 (1981), 487-494.

8. H. F. Trotter, Periodic automorphisms of groups and knots, Duke Math. J. 28 (1961), 553-558.

Department of Mathematics, University of Texas, Austin, Texas 78712

Current address: Department of Mathematics, Faculty of Science, The Australian National University, Canberra 2600, Australia 